\title{
On Technical Representation Research Suitable for Fast Design
}

\author{
Haijiao Sun \\ Zhuhai College of Jilin University \\ Zhuhai, China 519041
}

\begin{abstract}
Fast design generally refers to intensification of design creativity and representation training with high density in a short period of time. It makes for implementation of design creativity and formation of excellent product concept. What's more, it is an important means for modern products to get rid of the stale and bring forth the fresh in a short period of time and to occupy the market. Fast design focuses on cultivation of students' training and expression of combination of creative thought and practice. Through systematic study of design creativity, students can transform intangible creativity into knowable visual image. Thus, it can cultivate students' creative ability and expressive ability. During the process, the most important question is to "fast" express according to design idea. Then, we have to pick techniques and means suitable for "fast" expression among lots of techniques in order to provide better services for fast design. This paper will start with features of techniques in common use, select several techniques suitable for fast expression and make detailed research on pigment characteristics and drawing skills in order to improve quality and speed of fast design and lay solid foundation for teaching method and implementation policy of fast design.
\end{abstract}

\section{Keywords -suitable; fast design; technical representation}

\section{BRIEF DESCRIPTION OF FREQUENTLY-USED REPRESENTATION TECHNIQUES}

Expressive techniques of freehand sketching in most common use in design representation include: gouache technique, watercolor technique, spray painting technique, colored pencil technique, mark pen technique, chalk drawing technique etc. These techniques have their own advantages. Gouache technique has strong expressive force, full and thick colors, and strong covering power. It is easy to modify with deep depiction, therefore it is widely loved by people. Watercolor technique has clear, lucid and lively colors and its product modeling has clear structure outline. Spray painting technique has exquisite and rich pictures with even transition, subtle change and strong sense of reality. It has rather good effect on expression of glass, product photochromic change and reflection. And it has unique expressive force and modern sense.

However, we must be clear that, fast design requires using freehand sketching three view drawing and rough draft to rapidly and fully express design creativity and conception in limited time. This process is required to be "fast", so previously introduced gouache technique, watercolor technique and spray painting technique are not suitable for fast design. Because all these three techniques require mixing with water, all techniques using mixing with water have following requirements: Firstly, you need to mount the paper before drawing, otherwise the paper will wrinkle and uneven, influencing drawing effect. Secondly, there has to be a container for water, which is not speedy for fast design. Thirdly, according to painting requirements, there might be several times of coloring to obtain satisfactory effect. Especially that spray painting technique has complex drawing steps, so separation blade is used to repeatedly shield to complete. Therefore, seeing from time, steps, convenience and other factors, gouache technique, watercolor technique and spray painting technique are not suitable for fast design expression.

\section{ANALYSIS ON TECHNIQUES SUITABLE FOR FAST DESIGN}

Through above analysis, I think representation techniques suitable for fast design should satisfy the following conditions: The tool is convenient to carry, there is no need of mixing with water, there is no high requirement for paper and drawing steps are simple.

\section{A. Crayon Painting Techniques}

1) Characteristics of crayon painting: It is a kind of dry chalk made of pigment powder, which is called soft chalk in western countries. Generally, there are two kinds: round bar and square bar. There are also colorful chalks made of wood veneer. Their length is usually 8 to $10 \mathrm{~cm}$. Painting effect of colorful chalk can be as thick as ordinary oil painting. It also has smart feeling of watercolor with concise painting, and rich, gorgeous and elegant colors. It is unique in displaying delicate change and uniform color effect of metal and glass. Thus, it is widely loved by art lovers. It is totally different from traditional techniques in moulding and polishing. It does not need oil, water and other mediums to modulate color. It just needs mutual twisting between toner to obtain ideal color effect. It can replace spray painting to accomplish uniform color change.

2) Chalk drawing technique: Colorful chalk can be used to draw directly on paper. It is a kind of independent drawing form. Of course, it can be combined with other pigments to create special effect. Many painters like to use a small mount 
of colorful powder for bottoming and create a great tone atmosphere for painting. Then, they will use other tools, like gouache and mark pen, to conduct exquisite depiction. Toner is dry and unclear. Shallow color can directly cover deep color. Intuitive color contrast effect or transition effect can be created by spraying a light color on a deep color. By doing so, we can also create a uniform transition from the color of paper itself. Since pastels are soft, they are not suitable for drawing lines. Therefore, we can use carbon strip, colorful pencil or pencil to draw a draft. During blending process, you can use cloth, paper-made stump or finger to color. Cloth is mainly used to modulate the overall tone. Use rubbing of fingers to make colors change. Because we can control the force when modulating color with fingers, in addition, modulating with fingers can also control modulating range and avoid polluting surrounding colors.

Pastels have no special requirements for paper. You can choose paper with delicate or rough texture according to requirements of drawing. For example, if you want to create uniform and exquisite color changes, you need to choose smooth paper barely having textures. If you want to create special textures, you can choose a little rougher water paper.

It has to be mentioned that: Since toner is dry and it is easy to fall off due to long time, so you must use tailor-made oil painting liquid or transparent glass paper to protect the picture for long storage.

\section{B. Mark Pen Technique}

1) Characteristics of mark pen: Mark pen is widely used in oil-based type and water-based type. Oil pen has color of color printing and it can be used for drawing on any kind of surface. It is volatile with pungent smell. Water soluble pen has watercolor effects with characteristics of quick dry, no need of water mixing and convenient coloring. Therefore, it wastes no time to make preparations and clean. It is a kind of ready-made tool, which is suitable for quick expression of rough draft and sketch.

Mark pen point is square, round or sharp. Square head is suitable for large area and thick lines painting; Cusp is suitable for drawing hair stripe and slippery curve; Mark pen is volatile and it can be soaked in water under dry and stumbling color line circumstances. Of course, you can also use dry and rough brushwork to draw special texture effect.

2) Mark pen technique: When choosing color system, general priority is relative shallow and gray color, like warm gray and cold gray. The brighter the color is, the less it is used. Generally, divisions are highlights, highlight section, shade, projection and light and shadow junction. Therefore, when choosing colors, generally we choose 3 to 5 colors in each color system, namely color with high lightness, intermediate color and relative dark color.

Since width of frequently-used mark pen is limited to 3 to $6 \mathrm{~mm}$, it is not suitable for coloring of large area. Because slight stilted pen movement will lead to gap, which is impossible to make up for and makes direction in disorder. So we should try to avoid coloring of large area. If you have to conduct coloring of large area, please pay attention to direction of pen movement. Generally brushwork direction should focus on horizontal and vertical straightness, vanishing of brushwork toward vanishing point on vertical plane and $45^{\circ}$ brushwork principle. It also requires fast pen movement and concise brushwork without hesitation in order to ensure smooth and clean coloring with mark pen.

\section{Colored Pencil Technique}

Colored pencil is divided into water soluble and non water soluble type. Water soluble pencil is better in coloring and overlay of color and has better tinting strength on vegetable paper. Colored pencil has characteristics of convenient coloring, rich colors and powerful expressive force. It can use ivory board, vegetable paper, sketch paper and other ordinary papers to expressive the picture.

Due to excellence of colored pencil, it is not only suitable for rigorous painting, but also suitable for relaxed painting. However, as presentation drawing of product, it is impossible to waste long time to draw paintings as sketch or photo. If the work is just about simple coloring, it is usually simple without thickness or strong sense of layering. Therefore, colored pencil is usually combined with other drawing methods to achieve relative ideal effect.

\section{TeChnique Strategy OF FAST Design}

First, we should be clear that representation of fast design is to use various design concepts and design methods to accomplish required tasks of the topic quickly and accurately: like design positioning, creative rough draft, three view drawing, manual effect drawing etc. It mainly inspects expression of level of innovation of designing scheme, product modeling, framework combination, material texture and so on. Detailed introduction of technique strategy of fast design is displayed as follows with combination of an in-class case of the author. "Fig. 1"

\section{A. Problems to Think about before Fast Design}

The topic is children's enlightenment tools. Children's tools have a great variety and tools suitable for each age phase are different. Designers have to locate suitable age phase for their enlightenment tools, functions of enlightenment tools suitable for this age phase and special requirements for color, material, security and other factors of tools.

According to topic requirement and factors considered by us before designing, we can draw 3 to 5 design drafts based on our preliminary conception of design scheme and choose the most satisfactory scheme from them to draw three view drawing of product. Then, use representation techniques which can rapidly and accurately express design scheme to accomplish design works. It is also the most important part in fast design. 


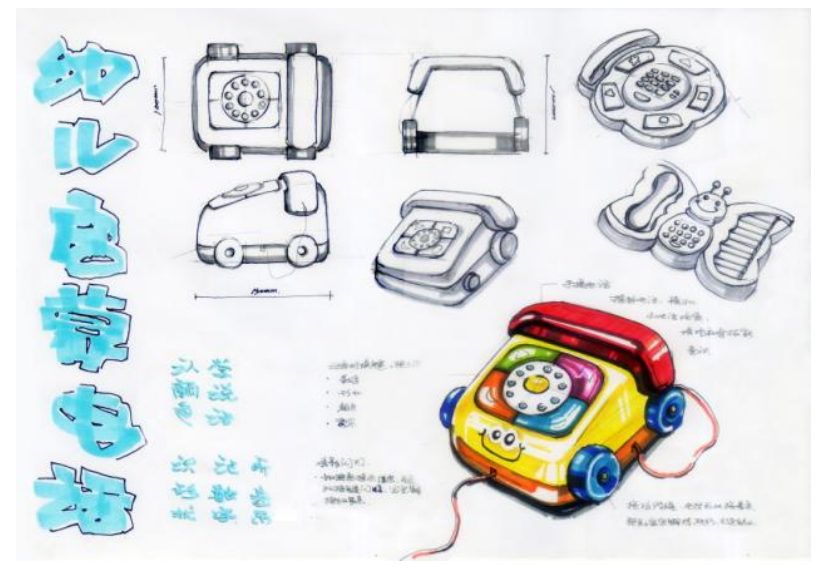

Fig. 1. Infant Enlightenment Phone Author: Sun Haijiao

\section{B. Drawing Steps of Techniques}

1) Drafting: Firstly, choose ways of perspective suitable for product expression to do drafting. If the designer has good painting skills, he/she can directly use a pen or a carbon pen to do drafting. If the designer is not skillful enough, he/she can use a pencil and focus on full expression of each structure. You should be clear about which part to take as the focus of expression and start with this part to depict and try to fully display products' light interception, shade and sense of texture. It is worth noting that: Relative long structure line can be accomplished with the aid of tools and try to directly draw short contour line, especially keys, logos and other small parts in order to avoid stiffness of painting.

2) Select a technique or multiple techniques according to content: Pay attention to the overall tone of picture, then consider partial color contrast and conduct detailed depiction after knowing application of the entire brushwork and change of details well. Do coloring from shallow to deep and try not to let color bleed out the contour line. If the painting needs a whole bottom color, pastels can be used for bottoming. Because colored pencils and mark pens are not suitable for coloring of large area in a short time. As to great tone, you need to pick intermediate color of the painting. Use wallpaper knife to peel off some pastel powder and directly use fingers or facial tissue or dry cloth to paint in order to do bottom coloring quickly or set off the atmosphere. There can be white space on illuminated face of the product. Please note that, if you use pens or carbon pens to draft directly, the advice is spreading toner before drafting, because pastels have certain covering power and will reduce clarity of chalk line.

Use mark pens to portray product object's projection and shade from shallow to deep. Since we have used pastels to do base coloring before this, we will emphasize on depicting of product's shady face, position of body twist, shadow line and so on next.

Use several colors shallower than intermediate color to paint illuminated face of product. Pay attention to transition of body, block and surface and changes of hook face. Draw another layer on boundary and use a color which is deeper than the original color to draw another layer at the same time. Repeat several times like this to increase the product's stereoscopic impression.

3) Material representation: There are various varieties of materials and textures of products, which can be roughly divided into four categories to express from the perspective of representation techniques.

\section{- Strong reflective material}

They are mainly stainless steel, mirror material, electroplating material and so on. Their characteristics are: relative strong light and shade contrast, existence of white space instead of drawing in highlights, emphasis on shade treatment, orderly and smooth brushwork, powerful line, a little color in highlights to make it more vivid and lifelike.

\section{- Half reflective material}

They are mainly plastic, marble and so on. Their characteristics are: relative gentle transition of black, white and gray, reflection weaker than metal, relative hard texture, richly changeable color and luster, need of a keynote in representation before using fine pens to sketch grain. In this case, children's enlightenment tools are mainly made of plastic, therefore please pay attention to uniform coloring and gentle transition.

\section{- Reflective and light-transparent material}

They are mainly glass, transparent plastic, organic glass and so on. You can directly do bottom coloring with the help of ambient color in representation and draw product's shape and thickness next. Then, emphasize on object's outline and light and shadow changes, and stress on highlight. Finally, deal with the reflective part.

\section{- Non-reflective and light-proof material}

They are divided into soft material and hard material. Soft materials mainly are fabric, sponge, leather products and so on; Hard materials mainly are wood, matte plastic, stone material and so on. When expressing soft material, you need to do coloring uniformly and moistly with smooth lines, gentle light and shadow contrast, and avoid using rigid lines and overemphasizing highlight. When expressing hard material, you should have clear distinctions between block and plane, clear structure, tall and straight and clear lines, and use dry brush to highlight grain result finally.

4) Painting adjustment and highlight treatment: Wholly grasp the painting's degree of balance and density relation, pay attention to color changes of object, and some ambient colors can also be considered. Brighten highlight of object at the same time, eraser can be used to scrub, and liquid opaque and modification pens can be used to draw highlight part if it is not bright enough. Highlight part should not be too bright. You can use colored pencils to draw a layer lightly to keep highlight from being too abrupt.

\section{Design Description}

Designer's general description of the whole design scheme: includes brief explanation of size, function, using steps and 
material texture of products and so on. It is also the designer's relative comprehensive interpretation of design scheme, comprehensively reflecting designer's cultural deposits, aesthetic accomplishment and understanding of product.

\section{REFERENCES}

[1] Jiang Yayu, Liu Shiwen. Methods and Approaches for Improvement of Students' Expressive Ability of Chinese Culture (2005. 3).

[2] Liao Huaying, Chen Feng. Research on the Expression Ability of Chinese Culture in English by Non-English Major Students (2011. 3).

[3] Hu Xiaorong. Research on the Expression Ability of Chinese Culture in English by English Major College Students (2015. 8).

[4] Luo Suqin, Li Xuan. Discussion on "Chinese Culture Aphasia" in English Teaching (2009. 6). 